International Mathematical Forum, 1, 2006, no. 1, 27-39

\title{
FINITE ILLUMINATION OF UNBOUNDED CLOSED CONVEX SETS
}

\author{
Mabel A. Rodríguez \\ Universidad Nacional de General Sarmiento \\ Instituto del Desarrollo Humano \\ Los Polvorines, Bs. As. ARGENTINA \\ mrodri@ungs.edu.ar \\ Fausto A. Toranzos \\ Universidad de Buenos Aires \\ Facultad de Ciencias Exactas y Naturales \\ ARGENTINA \\ fautor@sinectis.com.ar
}

\begin{abstract}
If $K$ is an unbounded closed convex subset of $\mathbf{E}^{d}$ having nonempty interior, we seek necessary and/or sufficient conditions to ensure that the boundary of $K$ can be externally illuminated from a finite set of directions. This problem was stated as open in a recent book by Boltyanski. The tools used in this search are those developed by Visibility Theory such as the ideas of star, inner stem, visibility cell, nova and cone of recession.
\end{abstract}

Mathematical Subject Classification: 52A30 - 52A20

Keywords: illumination, unbounded convex sets, external visibility

\section{Introduction}

Let $K$ be a convex body, i. e. a closed convex subset of the Euclidean $d$-space $\mathbf{E}^{d}$ having nonempty interior. We seek necessary and/or sufficient conditions in order that the whole boundary $\partial K$ can be externally illuminated from a finite number of directions. This problem is stated as open in [1]. At a first time we intend to relate the ideas of "illumination" and "external visibility" in order to use visibility tools to solve the problem. At a second time we aim 
to identify "critical" points in the boundary of $K$ whose illumination insures the illumination of most of $K$. We use some tools from cones and we present special boundary points associated to maximal boundary rays: the "tips" and "peaks" of $K$. Finally, following an approach already used in [2], we intend to concentrate the difficulties of illumination of $K$ into a bounded portion of it. To follow this way we introduce the "adequate cuts" of $K$. We find that if the set of all the peaks is bounded (or empty), then $K$ is finitely illuminable, so we destine the last part of this work to study, by means of a sequential approach, the case where the set of all the peaks of $K$ is unbounded.

\section{Preliminary Notes}

The open segment joining $x$ and $y$ is $(x, y)$, while the substitution of one or both parentheses by square ones indicates the adjunction of the corresponding endpoints. The open and the closed balls centered at $x$ and having radius $\delta$ are $U(x, \delta)$ and $B(x, \delta)$, respectively. If $K \subset \mathbf{E}^{d}$, its complement is $K^{C}$, its closure is $c l K$ and its interior is int $K$. The join of the sets $A$ and $B$ is the set

$$
J(A, B)=\bigcup\{[a, b] \mid a \in A, b \in B\} .
$$

In particular, $J(x, K)$ is the union of all the closed segments $[x, y]$ with $y \in K$. If $K$ is convex it holds $J(x, K)=\operatorname{conv}(\{x\} \cup K)$, where "conv" indicates the convex hull. We will denote with $\Omega_{d}$ the unit spherical surface of $\mathbf{E}^{d}$ and its elements will be called directions. We simbolize $R(x \rightarrow y)$ the closed ray issuing from $x$ and going through $y$. On the other hand, $R(y x \rightarrow)$ is the ray issuing from $x$ and going in the opposite direction of $R(x \rightarrow y)$. If $v$ is a direction, we will denote $R_{x}(v)=R(x \rightarrow x+v)$, i.e. the ray issuing from $x$ in the direction $v$. The set $K$ is a cone having apex $x$ if $\forall y \in K$ it holds $R(x \rightarrow y) \subset K$.

A convex component of $S$ is a maximal convex subset of $S$. We will say that $x$ sees $y$ via $S$ if $[x ; y] \subset S$. The star of $x$ in $S$ is the set $s t(x, S)$ of all the points of $S$ that see $x$ via $S$. A star-center of $S$ is a point $c \in S$ such that $s t(c, S)=S$. The mirador (convex kernel) of $S$ is the set mir $S$ of all the star-centers of $S$. $S$ is convex if mir $S=S$, and $S$ is starshaped if mir $S$ is not empty. We say that $x$ sees clearly $y$ via $S$ if $y$ admits a neighborhood $\mathcal{U}_{y}$ such that $x$ sees the whole $\left(\mathcal{U}_{y} \cap S\right)$ via $S$. The notion of clear visibility was introduced by N. Stavrakas in [3]. The nova (or clear star) of $p$ in $S$ is the set nova $(p, S)$ of all the points of $S$ that see $p$ clearly via $S$. The visibility cell of $p$ in $S$ is the set

$$
\text { vis }(p, S)=\{x \in S \mid \operatorname{st}(p, S) \subset \operatorname{st}(x, S)\} .
$$

Intuitively speaking, vis $(p, S)$ is the set of all points of $S$ that have better visibility than $p$. This idea was introduced by one of the present authors in 
[4]. If $y \in \partial S$ and $x \in \operatorname{st}(y, S)$ we say that the ray $R(x \rightarrow y)$ is inward through $y$ if there exists $t \in R(x y \rightarrow)$ such that $(y, t) \subset$ int $S$. Otherwise we say that $R(x \rightarrow y)$ is outward through $y$. If $y \in \partial S$, the inner stem of $y$ with respect to $S$ is the set ins $(y, S)$ formed by $y$ and all the points of $\operatorname{st}(y, S)$ that issue outward rays through $y$.

A direction of recession of $K$ is an element $v \in \Omega_{d}$ such that for every $x \in K$ the halfline $R(x \rightarrow x+v)$ is included in $K$. The set of all the directions of recession of $K$ is denoted $D(K)$ and the cone of recession of $K$ (denoted $R(K))$ is the union of all the rays issuing from the origin and directed by the elements of $D(K)$. For a thorough study of this cone we refer to [5]. The main properties of unbounded convex sets are the following statements whose well known proofs are not included here.

Theorem 2.1 Let $K$ be an unbounded closed convex set and $R_{x}(v)$ a ray included in $K$. Then, $\forall z \in K$ the parallel ray $R_{z}(v)$ is also included in $K$.

Theorem 2.2 If $K$ is an unbounded closed convex set, then $D(K)$ and $R(K)$ are nontrivial.

Following the terminology introduced by Boltyanski we state the next definition.

Definition 2.3 We say that the point $x \in \partial S$ is illuminated by the direction $v$ if the ray $R_{x}(v)$ passes through interior points of $K$. An illumination of $K$ is a set of directions such that every boundary point of $K$ is illuminated by some element of it. The set $K$ is finitelly illuminable (abbr. $K$ is f.i.) if it admits a finite illumination.

\section{Main Results}

The following series of results are referred to the visibility in the closure of the complement of the convex body that we intend to illuminate. As we will see, external visibility of a set gives information about its possibilities of illumination.

Lemma 3.1 Let $K$ be a convex body and denote $S=\operatorname{cl} K^{C}$. Let $M$ be a convex component of $S$. Then $M$ is a closed halfspace.

Proof. It is clear that $S \cap i n t K=\emptyset$, and a fortiori $M \cap i n t K=\emptyset$. Hence, a standard separation theorem yields a hyperplane $H$ that define two closed halfspaces $H^{+}$and $H^{-}$such that $M \subset H^{+}$and $K \subset H^{-}$. Furthermore, from the construction it is clear that $H^{+} \subset S$. Hence, from the maximality of $M$ it follows that $M=H^{+}$. 
Remark. A certain type of converse statement of this lemma is valid, namely: "Let $K$ be a closed set with nonempty interior, such that every convex component of $S=c l K^{C}$ is a closed halfspace. Then $K$ is a convex body". We leave the proof to the reader.

Lemma 3.2 Let $K$ be a convex body and denote $S=$ cl $K^{C}$. If $x \in \partial K=$ $\partial S$, then ins $(x, S)=\bigcup_{y \in K} R(y x \rightarrow)$.

Proof. Let us denote $\widehat{C}(x, K)=\bigcup_{y \in K} R(y x \rightarrow)$ (and we will merely write $\widehat{C}$ if there is no doubt about $x$ and $K)$ and $C(x, K)$ the conic hull of $K$ having vertex at $x$ (i.e. the union of all the rays issuing from $x$ and going through points of $K)$. Notice that $\widehat{C}(x, K)$ is the symmetrical cone of $C(x, K)$ with respect to $x$.

1. Let $w \in \widehat{C}$, then $w=-v+x$ for some $v \in C(x, K)-\{x\}$. Hence there must exist $a \in K$ such that $v+x \in R(x \rightarrow a)$. It is clear that the points $x, v+x$ and $-v+x$ are aligned. Furthermore, by construction, also the point $a$ is in the same line with these three points. From the convexity of $K$ it follows $[x, a] \subset K$, whence $\forall t \in R(w x \rightarrow)$ it holds $[t, x] \cap$ int $S=\emptyset$. This means that the ray $R(w \rightarrow x)$ is outward (from $S)$ through $x$. Hence $w \in$ ins $(x, S)$.

2. Conversely, assume that $w \in$ ins $(x, S)$, hence $R(w \rightarrow x)$ must be $S$ outward through $x$ and there must exist $t \in R(w x \rightarrow)$ such that $(t, x) \cap$ $K^{C}=\emptyset$. Hence, $t \in K$ and clearly $w \in R(t x \rightarrow)$, whence $w \in \widehat{C}$.

This completes the proof of the lemma.

Theorem 3.3 Let $K$ be a convex body and $S=$ cl $K^{C}$. Take $x \in \partial S$ and denote $A=$ int ins $(x, S), B=$ int nova $(x, S), C=$ int vis $(x, S), D=$ int $\widehat{C}(x, K)$ (as defined in the previous lemma). Then these four sets coincide.

Proof. We split the proof into five independent steps:

1. $A \supset B$. It has been proved [4] that in this case holds nova $(x, S) \subset$ ins $(x, S)$.

2. $A \subset B$. We prove that int ins $(x, S) \subset \operatorname{nova}(x, S)$ and the thesis follows. Let $t \in A$, then $\exists \varepsilon>0$ such that $U(t, \varepsilon) \subset$ ins $(x, S)$. We split this case in three assumptions: a) $R(t x \rightarrow) \cap i n t K \neq \emptyset$, otherwise it would exist $H$ a support hyperplane of $K$ such that $R(t x \rightarrow) \subset H$. We denote $H^{+}$ the closed half-space determined by $H$ such that contains $K$. Then int $H^{+} \cap U(t, \varepsilon) \neq \emptyset$ and these points of $U(t, \varepsilon)$ do not produce outward rays 
through $x$ which is a contradiction. Let us take $w \in R(t x \rightarrow) \cap i n t K$ and $U=U(w, \varepsilon) \subset \operatorname{int} K$. We denote $J=J(U, t)$ and $M=J \cap \partial K$. Notice that every point $y \in M$ satisfies $y \in\left(t, w^{\prime}\right)$ with $w^{\prime} \in U \subset$ int $K$. The second assumption states b) $t$ sees $y, \forall y \in M$. Otherwise there would exist $y \in M$ such that $t$ does not see $y$, then there exists $w^{\prime} \in U \subset$ int $K$ such that $y \in\left(t, w^{\prime}\right)$. If $t$ does not see $y$, there would exist $k \in(t, y) \cap K$ and then $k \in K, w^{\prime} \in$ int $K$ which implies, by means of Accessibility Theorem, $\left(w^{\prime}, k\right) \subset$ int $K$ and $y \in\left(w^{\prime}, k\right)$ which contradicts the fact that $y \in \partial K$. The last part states that c) if $\delta>0$ is such that $U(x, \delta) \subset J$ then $t$ sees $U(x, \delta) \cap S$ and its proof is analogous to b).

3. $A \supset C$. Let $y \in C$, then there exists $\delta>0$ such that $U=U(y, \delta) \subset$ vis $(x, S)$. If $R(y \rightarrow x)$ would be $S$-inward through $x$, there would exist $t \in R(y x \rightarrow)$ such that $(x, t) \subset$ int $S=K^{C}$. If we pick $z \in(x, t)$ it is clear that $x$ would see $z$ via $S$, whence every point of $U$ would see $z$ via $S$. This would imply that $J=J(z, U)$ would be included into $S$. But this inclusion would yield that $x \in i n t S$, in contradiction with our selection of $x$ as a point of $\partial S$. This contradiction stems from the assumption that $R(y \rightarrow x)$ be inward. Hence it must be $S$-outward through $x$, and $y \in$ ins $(x, S)$. The inclusion $A \supset C$ follows immediately.

4. $C \supset D$. If $H$ is a support hyperplane of $K$ through $x$, then it clearly separates $D=\operatorname{int} \widehat{C}$ from int $K$. Let $M$ be a convex component of $S$ such that $x \in M$. From Lemma 3.1 we know that $M$ is a closed halfspace determined by a support hyperplane through $x$. Hence int $\widehat{C}$ is included in any such $M$. But it has been proved (see [4]) that vis $(x, S)$ is the intersection of all the convex components of $S$ that include $x$. This means that $D \subset$ vis $(x, S)$, whence $D \subset C$.

5. $A=D$. This follows inmediately from the previous lemma.

The conjunction of these statements yields the thesis.

Lemma 3.4 Let $K$ be a convex body, $S=c l K^{C}$, and $x \in \partial S$. Then both ins $(x, S)$ and nova $(x, S)$ are convex sets. Furthermore, ins $(x, S)$ is a convex cone having $x$ as an apex.

Proof. It is known (see [4]) that vis $(x, S)$ is always convex. The first statement of the corollary follows from the previous lemma. The second statement is a consequence of Lemma 3.2.

Theorem 3.5 Let $K$ be a convex body, $S=c l K^{C}$, and $x \in \partial S$. The point $x$ is illuminated by the direction $v$ if and only if $\exists y \in$ int ins $(x, S)$ such that $v=x-y$. 
Proof. $(\Longrightarrow)$ There must exist $p \in R_{x}(v) \cap$ int $K$. Denote $L$ the line through $x$ and $p$ and $R=R(p x \rightarrow)=R_{x}(-v)$. We claim that

$$
\{y \in R \mid y \neq x\} \subset \text { intins }(x, S) \text {. }
$$

From the well known Linear Accesibility Theorem for convex bodies follows that

$$
(p, x) \subset \operatorname{int} K
$$

Furthermore, if we pick a generic point $y \in R$ and different from $x$, it follows that $y \in K^{C}=$ int $S$ for otherwise the just quoted theorem would yield $x \in$ int $K$, a contradiction. Hence it holds $(y, x) \subset$ int $S$. Moreover, there exists $\delta>0$ such that $U_{p}=U(p, \delta) \subset K$. Denote $h$ the homothetical mapping centered at $x$ that maps $p$ into $y$. Then there exists $\varepsilon>0$ such that $U_{y}=$ $U(y, \varepsilon)=h\left(U_{p}\right)$. Using the same argument as above, it follows that

$$
U_{y} \subset \operatorname{ins}(x, S)
$$

From (1) and (2) follows our claim. It just remains to pick $y=x-v \in R$.

$(\Longleftarrow)$ Let $y \in$ intins $(x, S)$ then there exists $t \in R(y x \rightarrow) \cap S^{c}=R(y x \rightarrow$ )$\cap$ int $K$. Then $t$ belongs to $L$, the line going through $x$ and $y$. So it holds that $v=\frac{x-y}{\|x-y\|}$ is the direction of $L$ and as we know that $t \in L \cap i n t K$ it results that this direction $v$ defined in this way illuminates $x \in \partial K$.

Until otherwise stated $K$ is a closed unbounded convex set having nonempty interior and we aim to identify "critical" points in the boundary $\partial K$, whose illumination insures the illumination of most of $K$.

The next two results show us that the case where $K$ includes lines can be discarded, and that the significative case is the illumination of unbounded but line-free convex bodies.

Lemma 3.6 Let $K$ be a closed convex set that includes lines. Then there exists a unique linear subspace $L$ and a closed convex set $T$ that is line-free, such that $K=T \oplus L$.

Proof. See Proposition 4 of Paragraph 2.5 of [6].

Lemma 3.7 Let $K$ be a closed convex set that includes lines and let $K=$ $T \oplus L$ the decomposition provided by the previous lemma. Then any illumination of $T$ is also an illumination of $K$.

Proof. Let $L^{\perp}$ be a linear subspace complementary to $L$ such that $T \subset L^{\perp}$, and let $x \in \partial K$. Hence $x=t+l$ with $t \in \partial T$ and $l \in L$. Assume that the direction $v$ illuminates $t$ as a point of $T \subset L^{\perp}$. Hence there must exist $\lambda_{0}>0$ 
such that $p=\lambda_{0} v+t \in$ int $T$ (in the relative topology of $L^{\perp}$ ). This clearly implies that

$$
p+l=\lambda_{0} v+t+l=\lambda_{0} v+x \in \operatorname{int} K
$$

whence $v$ illuminates $x$ as a point of $\partial K$.

Hence, for unbounded closed convex and line-free sets, the new fact is the existence of directions of recession. These directions can illuminate most points of the boundary, but not all of them, as we will see. A maximal boundary ray of $K$ is a maximal halfline included into $\partial K$. In this definition, the word "maximal" means that the halfline is not properly included in other halfline with the same property. A tip of $K$ is the initial point of a maximal boundary ray of $K$, and a peak of $K$ is a tip of $K$ that is also an extremal point of $K$ (i.e. the peak is not included in an open segment whose endpoints lay in $K$ ). Consider the set $K \subset \mathbf{E}^{3}$ defined by

$$
K=\{(x, y, z) \mid x \geq 0 ; 1 \geq y \geq 0 ; z \geq 0\} .
$$

The points $\theta=(0,0,0)$ and $p=(0,1,0)$ are peaks of $K$, while any other point of the open segment $(\theta ; p)$ is a tip but not a peak of $K$.

Lemma 3.8 Let $R$ be a maximal boundary ray of $K$ and $x$ its tip. Then any direction that illuminates $x$ also illuminates any other point of $R$.

Proof. Let $R=R_{x}(u)$ be a boundary ray, $v \in \Omega_{d}$ a direction that illuminates $x$, and $y \in R$ but different from its tip. There must exist $t \in R_{x}(v)$ and $\delta>0$ such that $U(t, \delta) \subset$ int $K$. Since $R_{x}(u) \subset K$ it follows from Theorem 2.1 that $R_{t}(u) \subset K$. Furthermore, we claim that $R_{t}(u) \subset$ int $K$. Let $z$ be a generic point of $R_{t}(u)$ different from $t$. Denote $z^{\prime}=z+u$ and $J=J\left(z^{\prime}, U(t, \delta)\right)$. It is very easy to verify that $z \in$ int $J \subset$ int $K$ and this proves our claim. Since $y \in R_{x}(u)$ there exists $\lambda>0$ such that $y=x+\lambda u$. Denote $t^{\prime}=t+\lambda u$. The fact that $t \in R_{x}(v)$ implies that $t^{\prime} \in R_{y}(v)$ and, as we have just seen, $t^{\prime} \in \operatorname{int} K$. Hence $v$ illuminates $y$.

Lemma 3.9 Let $v \in D(K)$ and $x \in \partial K$ such that the ray $R(x \rightarrow x+v)$ is not a boundary ray of $K$. Then $v$ illuminates $x$.

Proof. It is clear that $R_{x}(v) \subset K$ and by hypothesis this ray is not entirely included in $\partial K$. By the same argument used in the previous lemma, it follows that every $y \in R_{x}(v)$ different from $x$ is an interior point of $K$. This implies clearly that $v$ illuminates $x$.

Lemma 3.10 Let $I \subset \Omega_{d}$ be a set of directions that illuminates every peak of $K$, and let $v \in D(K)$. Then $\widehat{I}=I \cup\{v\}$ is an illumination of $K$. 
Proof. Let $x$ be a generic point of $\partial K$. Without loss of generality, we may assume that $x$ is not a peak, since the illumination of peaks is accomplished by the elements of $I$. Furthermore, if $x$ is not included in a boundary ray, Lemma 3.9 assures that $v$ illuminates it. Hence, suppose that $x \in R$, a maximal boundary ray. Moreover, assume that $x$ is the tip of $R=R_{x}(u)$. From our first assumption, it follows the existence of two points $a$ and $b$ in $K$ such that $x \in(a, b) \subset K$. Hence there must exist a maximal linear element $L$ (either an interval or a halfline) such that $(a, b) \subset L \subset K$. Denote $p$ an extreme point of $L$. We claim that $p$ is a peak of $K$. Otherwise, there would exist an open interval $(c, e)$ such that $p \in(c, e) \subset K$. Denote $H$ a support hyperplane of $K$ through $x$ that includes $R$ and $L$, and let $H^{+}$be the closed halfspace bounded by $H$ and including $K$. It is clear, from the construction, that $p \in H$, and this would imply immediately that $(c, e) \subset H$. Denote $T_{a}=\operatorname{conv}\{a ; c ; e\}$ and $T_{b}=\operatorname{conv}\{b ; c ; e\}$. It is clear that one of these triangles would be included in the other, so call $T$ the largest one. Since clearly $T \subset K$ and $x \in \operatorname{rel}$ int $T$, this would contradict the maximality of the boundary ray $R_{x}(u)$. Hence $p$ must be a peak and some $w \in I$ illuminates it. This means that there exists $t \in R_{p}(w) \cap$ int $K$. Assume that $a$ is the farthest point (from the pair $\{a, b\}$ ) to $p$, take $\delta>0$ such that $U(t, \delta) \subset K$ and denote $J=J(a, U(t, \delta)) \sim\{a\}$. Then, clearly $J \subset$ int $K$ and $J \cap R_{x}(w)$ is not empty. Hence, $w$ also illuminates $x$. It remains to consider the case where $x \in R$, a boundary ray, but $x$ is not the tip of $R$. Then $R=R_{z}(u)$ with $z$ different from $x$. From our last argument it follows that there must exist $w \in I$ that illuminates $z$. Lemma 3.8 implies that $w$ also illuminates $x$.

Example: Let us consider $K=\left\{(x, y) \in E^{2} \mid 0 \leq x \leq 1,0 \leq y\right\}$. Points $(0,0) \mathrm{y}(1,0)$ are the only peaks of $K$, and the directions $v=\left(\frac{1}{\sqrt{2}}, \frac{1}{\sqrt{2}}\right)$ and $w=\left(-\frac{1}{\sqrt{2}}, \frac{1}{\sqrt{2}}\right)$ illuminate each of them, respectively. The only direction of recession of $K$ is $u=(0,1)$, but the set $I=\{v, w\}$ illuminates $K$. Hence, the illumination $\widehat{I}$ proposed by the previous result may not be minimal.

Corollary. Let $K \subset E^{2}$ be closed, convex, unbounded, line-free and with nonempty interior. Then $K$ admits an illumination by, at most, three directions.

Proof. If $K$ admits no peaks, Lemma 3.9 implies that a single direction of recession illuminates it. We intend to show that $K$ has, at most, two peaks. On the contrary, assume that $p, q, r$ are distinct peaks of $K$. Denote $R_{p}, R_{q}$ and $R_{r}$ the maximal boundary rays that these points would determine, and $L_{p}, L_{q}, L_{r}$ the lines that would include these rays. It is clear that each of these lines would be a support line of $K . L_{p}^{+}$would denote the closed halfplane bounded by $L_{p}$ and including $K$, the same for $L_{q}^{+}$and $L_{r}^{+}$. Then $S=L_{p}^{+} \cap L_{q}^{+}$ 
would be a stripe of the plane or a planar angle, and $K \subset S$. Hence it would hold $r \in$ int $S$ and $L_{r}$ would pass through interior points of $K$, in contradiction with its condition of support line. This contradiction is originated by the assumption of the existence of three peaks. If $K$ has one or two peaks, an illumination of $K$ can be obtained by a direction of illumination for each peak and a direction of recession of $K$.

Lemma 3.11 If there exists $v \in D(K)$ such that no boundary ray of $K$ has direction $v$, then $\{v\}$ is an illumination of $K$.

Proof. Pick a generic point $x \in \partial K$ and denote $R=R_{x}(v)$. By hypothesis $R$ is not included in $\partial K$ and $R \subset K$. This means that $R$ includes interior points of $K$, whence $x$ is illuminated by $v$.

Corollary. Let $v \in$ int $D(K)$ (in the relative topology of $\Omega_{d}$ ). Then $\{v\}$ is an illumination of $K$.

Proof. Denote $\widetilde{R}_{z}(u)$ the cone $R_{z}(u)$ with its tip $z$ removed. The hypothesis implies that $\widetilde{R}_{\theta}(v) \subset$ int $R(K)$, the cone of recession of $K$. Let $x$ be a generic point of $\partial K$. Then $\widetilde{R}_{x}(v) \subset x+$ int $R(K) \subset$ int $K$. Hence $v$ illuminates $x$.

Until otherwise stated, $K$ denotes an unbounded but line-free convex body in $\mathbf{E}^{d}$. Following an approach already used in [2], we intend to concentrate the difficulties of illumination of $K$ into a bounded portion of it.

Definition 3.12 Let $H$ be a hyperplane that includes interior points of $K$, and let $H^{+}$and $H^{-}$the closed halfspaces determined by $H$. Denote $K^{+}=$ $K \cap H^{+}$and $K^{-}=K \cap H^{-}$, both closed and convex. The pair $\left\{K^{+} ; K^{-}\right\}$is an adequate cut of $K$ if it holds:

- $K^{+}$is bounded and $K^{-}$is not.

- No peak of $K$ is included in $K^{-}$.

Lemma 3.13 If $K$ admits an adequate cut, it is finitely illuminable.

Proof. The set $P(K)$ of all the peaks of $K$ (that can be empty) is included in $K^{+}$that is compact, hence finitely illuminable (see[1]). This implies that there is a finite set of directions that illuminate all the elements of $P(K)$. Lemma 3.10 yields the thesis.

Theorem 3.14 If the set of all the peaks of $K$ is bounded (or empty), $K$ is finitely illuminable. 
Proof. It is easy to obtain an adequate cut of such a set, whence the previous lemma implies the theorem.

Example: A polyhedral set, having finitely many peaks, or an elliptic paraboloid, with no peaks, are examples of application of Theorem 3.14.

Until otherwise stated, $K$ is a convex body in $\mathbf{E}^{d}$. In accordance with the statement of Theorem 3.14, here we concentrate our efforts on the case where the set of all the peaks of $K$ is unbounded.

Lemma 3.15 Assume there exists a sequence $\left\{x_{n}\right\}$ in $\partial K$ such that $\forall v \in$ $\Omega_{d}, v$ illuminates at most finitely many terms of the sequence. Then $K$ is not $f i$.

Proof. Assume that $K$ would be fi. This would imply that each of the finite directions of illumination would illuminate a finite number of terms. The union of finitely many finite sets is finite, and the sequence has infinitely many terms, a contradiction.

Let us define the relation " $v$ illuminates $x$ " as a relation from $\Omega_{d}$ to $\partial K$ . Clearly, it is a relation "many to many".

Lemma 3.16 The relation " $v$ illuminates $x$ " is doubly continuous in the following sense:

1. If $v$ illuminates $x$ and $x^{\prime}$ is close enough to $x$, then $v$ illuminates $x^{\prime}$.

2. If $v$ illuminates $x$ and $w$ is close enough to $v$, then $w$ illuminates $x$.

Proof. (1) $v$ illuminates $x$ implies there exists $\lambda>0$ such that $t=x+\lambda v \in$ int $K$. This means that there exists $\delta>0$ such that the open ball $U(t, \delta) \subset K$. Denote $\widehat{U}=U(x, \delta) \cap \partial K$. It is clear that $\widehat{U}$ is a neighborhood of $x$ in the relative topology of $\partial K$. Furthermore, it is easy to verify that $\forall x^{\prime} \in \widehat{U}$ it holds that $v$ illuminates $x^{\prime}$.

(2) Once more, if $v$ illuminates $x$ there exists $\lambda>0$ such that $t=x+\lambda v \in$ int $K$, and $\delta>0$ such that $U(t, \delta) \subset K$. For each $p \in U(t, \delta)$ we associate $\widetilde{p}=\frac{p-x}{\|p-x\|}$. Notice that each $\widetilde{p}$ is an unit vector, and the set $\widetilde{U}=\{\widetilde{p} \mid p \in U(t, \delta)\}$ is a neigborhood of $v$ in the relative topology of $\Omega_{d}$. Furthermore, if $w \in \widetilde{U}$ then $w$ illuminates $x$.

Lemma 3.17 Let $\left\{x_{n}\right\}$ be a sequence in $\partial K$ such that $\forall v \in \Omega_{d}$, v illuminates finitely many terms of it. Then $\left\{x_{n}\right\}$ is unbounded. Furthermore, the sequence of directions $v_{n}=\frac{x_{n}-x_{1}}{\left\|x_{n}-x_{1}\right\|}$ converges towards a direction of recession of $K$. 
Proof. First assume that $\left\{x_{n}\right\}$ is convergent to a point $x_{o}$ and pick a direction $v_{o}$ such that $v_{o}$ illuminates $x_{o}$. Since $x_{o}=\lim x_{n}$ it follows from Part 1 of Lemma 3.16, that $v_{o}$ would illuminate infinitely many terms of the sequence, a contradiction. If we only assume boundedness of $\left\{x_{n}\right\}$, it would admit a convergent subsequence with the same contradiction. For the second statement of this lemma, define $p_{v}$ as the second intersection of the ray $R\left(x_{1} \rightarrow x_{1}+v\right)$ with $\partial K$ and $\lambda(v)=\left\|x_{1}-p_{v}\right\|$ where this last function is defined for every $v \in \Omega_{d}$ such that $p_{v}$ exists. This function is usually called radial function of $K$ with center $x_{1}$, and is known to be continuous. Let $v_{o}$ be a point of accumulation of the sequence $\left\{v_{n}\right\}$. In any neighborhood of $v_{o}$ there must be infinitely many directions $v_{n}$ having $\lambda\left(v_{n}\right)$ arbitrarily large. Hence, the point $p_{v_{o}}$ cannot exist, and the ray $R\left(x_{1} \rightarrow x_{1}+v_{o}\right)$ lies inside $K$.

In the sequel, we keep the notation $P(K)$ for the set of all the peaks of $K$, and denote $S=c l\left(K^{C}\right)$.

Theorem 3.18 If $P(K)$ is unbounded and $K$ is not fi there must exist an unbounded sequence $\left\{x_{n}\right\} \subset P(K)$ such that

$$
\bigcap_{n \in N}\left[\operatorname{int}\left(\operatorname{ins}\left(x_{n}, S\right)\right)-x_{n}\right]=\emptyset
$$

Proof. We will prove the contrapositive statement, i.e.:

Assume that every unbounded sequence $\left\{x_{n}\right\}$ of peaks of $K$ verifies $\bigcap_{n \in N}\left[\operatorname{int}\left(\operatorname{ins}\left(x_{n}, S\right)\right)-x_{n}\right]$ not empty. Then $K$ is $f$.

Let $\left\{x_{n}\right\}$ be any sequence in $\partial K$ such that $\bigcap_{n \in N}\left[\operatorname{int}\left(\operatorname{ins}\left(x_{n}, S\right)\right)-x_{n}\right] \neq \emptyset$. We claim the existence of a direction that illuminates the whole sequence. Take $u \in \bigcap_{n \in N}\left[\operatorname{int}\left(\operatorname{ins}\left(x_{n}, S\right)\right)-x_{n}\right]$. Then it holds

$$
\forall n u+x_{n} \in \operatorname{int}\left(\operatorname{ins}\left(x_{n}, S\right)\right)
$$

From Theorem 3.5 it follows that $-u=x_{n}-\left(u+x_{n}\right)$ illuminates every term of the sequence. Now consider a countable set $D=\left\{p_{n}\right\}_{n \in N}$ dense in $P(K)$. The sequence $\left\{p_{n}\right\}$ is unbounded by hypothesis, hence there must exist a direction $v_{o}$ that illuminates every point of $D$. Part 1 of Lemma 3.16 yields that $v_{o}$ illuminates every point of $P(K)$. If $v$ is any direction of recession of $K$, it follows from Lemma 3.10 that the set $\left\{v ; v_{o}\right\}$ is an illumination of $K$.

The condition of the last theorem is too strong to be a characterization of fi sets, since it implies that $K$ admits an illumination formed by only two directions, hence the converse implication results false. The following counterexample shows this falsehood. 
Example: Define $M=\left\{(x, y) \in \mathbf{E}^{2} \mid y \geq x^{2}\right\}$ and $K=M \times[0,1] \subset \mathbf{E}^{3}$. It is easy to see that $K$ is fi. Nevertheless, we can construct an unbounded sequence $\left\{p_{n}\right\} \subset P(K)$ where

$$
p_{n}= \begin{cases}\left(n, n^{2}, 0\right) & \text { if } n \text { is even } \\ \left(-n, n^{2}, 0\right) & \text { if } n \text { is odd }\end{cases}
$$

It is clear that for such a sequence it holds

$$
\bigcap_{n \in N}\left[\operatorname{int}\left(\operatorname{ins}\left(p_{n}, S\right)\right)-p_{n}\right]=\emptyset
$$

Considering the previous theorem and example, we formulate the following conjecture.

Conjecture. Assume that $P(K)$ is unbounded. $K$ is $f i$ if and only if every unbounded sequence of peaks $\left\{x_{n}\right\}$ admits a subsequence $\left\{x_{n_{k}}\right\}$ such that $\bigcap_{k}\left[\operatorname{int}\left(\right.\right.$ ins $\left.\left.\left(x_{n_{k}}, S\right)\right)-x_{n_{k}}\right]$ is not empty.

Unfortunately, up to now we have been able to prove only one of the implications of this statement.

Lemma 3.19 Let $K$ be $f i$ and such that $P(K)$ is unbounded. Then any unbounded sequence $\left\{x_{n}\right\}$ of peaks admits a subsequence $\left\{x_{n_{k}}\right\}$ such that $\bigcap_{k}\left[\operatorname{int}\left(\operatorname{ins}\left(x_{n_{k}}, S\right)\right)-x_{n_{k}}\right]$ is not empty.

Proof. Let $\left\{x_{n}\right\} \subset P(K)$ be unbounded. Lemma 3.15 yields a direction $v$ that illuminates infinitely many terms of the sequence, say the subsequence $\left\{x_{n_{k}}\right\}$. Since $v$ illuminates $x_{n_{k}}$ for every index $k$, by Theorem 3.5 there must exist $y \in$ int $\left(\right.$ ins $\left.\left(x_{n_{k}}, S\right)\right)$ such that $v=x_{n_{k}}-y$. This implies that $-v \in$ $\bigcap_{k}\left[\operatorname{int}\left(\operatorname{ins}\left(x_{n_{k}}, S\right)\right)-x_{n_{k}}\right]$.

ACKNOWLEDGEMENTS. We want to acknowledge Dra. Ana Forte Cunto for her helpful ideas in the proof of Theorem 3.3 and Lic. Alberto Formica for his suggestions after a carefull reading of this paper.

\section{References}

[1] V. Boltyanski, H. Martini and P.S. Soltan, Excursions into Combinatorial Geometry, Springer, (1997).

[2] G.L. Hansen and F.A. Toranzos, Polyhedron = Polytope - 1 Facet, New Zealand J. of Math., 28 (1999), 203-206. 
[3] N.M. Stavrakas, The dimension of the convex kernel and points of local nonconvexity, Proc. Amer. Math. Soc., 34 (1972), 222-224.

[4] F.A. Toranzos, Critical visibility and outward rays, J. Geom. 33 (1988), 155-167.

[5] F. Jongmans, Etude de cônes associés à un ensemble, Université de Liège (mimeographed notes), Liège, 1980-1981.

[6] B. Grünbaum, Convex Polytopes, Interscience, London, 1967.

Received: March 1, 2001 